\title{
Improvement in Oxidative Stability of Soybean Oil by Mango Kernel Extracts
}

\author{
Ambati Padmaja and Nunna B. L. Prasad \\ J.N.T.U.A. Oil Technological Research Institute, Anantapur 515001 (A.P.), India
}

Received: August 3, 2011 / Published: February 20, 2012.

\begin{abstract}
This work was undertaken to explore the potential of fruit waste materials as sources of natural antioxidants. Kernels of four varieties of mangoes of Indian origin were studied. Kernels were extracted with five different solvents, tested for extraction efficiency and total phenolic content (TPC). As the methanol proved to be the best solvent, it was used for further analysis. The methanolic extracts of kernels had reducing power (RP) and scavenged 2, 2 diphenyl-1-picryl hydrazyl (DPPH) radical, which is related to their antioxidant activity (AA). When analyzed in HPLC, the extracts showed the presence of phenolic compounds. The extracts had the higher capacity to reduce the formation of peroxides and slow down the rate of oxidation than butylated hydroxyl toluene (BHT) in refined, bleached deodorized soybean oil (RBD SBO). Based on the results obtained mango kernels are potential source of natural antioxidants owing to their antioxidant activity.
\end{abstract}

Key words: Mango kernels, soybean oil (RBD SBO), Gallic acid, 2, 2 diphenyl-1-picryl hydrazyl (DPPH) activity.

\section{Introduction}

Antioxidants are compounds that when added to food products, especially to lipids and lipid containing foods can increase the shelf-life by retarding the process of lipid peroxidation, which is one of the major reasons for the deterioration of food products during processing and storage [1]. Limitations associated with the use of synthetic antioxidants have stimulated screening of various edible plant materials in search for harmless, effective and acceptable additives to enhance the shelf life of food products. High consumption of fruits and vegetables has been associated with a lower incidence of degenerative diseases. Such protective effects are thought to be partially associated with various antioxidant compounds contained in these foods [2]. Antioxidant activity (AA) in fruits varies among species and cultivars. Diverse antioxidant compounds present in fruits are responsible for high antioxidant capacity [3].

Corresponding author: Nunna B. L. Prasad, research officer, research field: lipid science \& technology. E-mail: Prasad_nbl@yahoo.com.
The primary waste fractions which are peel and seed residues contain high amounts of bioactive components that can be potentially exploited as antioxidant agents and nutraceuticals. Recently, a growing interest in substitution of synthetic antioxidants by natural ones has fostered research on screening of plant materials, especially from inexpensive or residual sources from agricultural industries for evaluating new antioxidants [4].

Mango (Mangifera indica L. Anacardiaceae) is one of the most important tropical fruits. India ranks $1^{\text {st }}$ in its production of mangoes, puree, nectar, leather, chutney, canned slice in the world and experiences world wide popularity. Major byproducts of mango processing are peels and seeds amounting for $20 \%$ and $25 \%$ of total fruit weight respectively. Because these byproducts represent serious disposal problems various attempts at utilizing seed kernels and peels have been made in past few decades. Number of investigations has been conducted on composition and utilization of kernels [5-7]. The purpose of this study was to investigate the total phenolic content (TPC) of the 
mango varieties under study by using various organic solvents, to know the antioxidant activity of the mango kernel extracts and its role in prevention of rancidity in RBD SBO.

\section{Materials and Methods}

Baneesha, malgoa, totapuri and neelam variety mango fruits were purchased from local market and were identified by botany dept. of local university. Antioxidant-free RBD SBO was procured from M/s Cargill Foods India Ltd, Pune, India. These oil samples were stored in amber coloured bottles. Gallic acid was purchased from Sigma. Solvents and other chemicals were purchased from Himedia and Sd. fine.

\subsection{Sample Preparation}

The seeds of four different mango varieties baneesha kernel (BK), malgoa kernel (MK), totapuri kernel (TK) and neelam kernel (NK) were separated from fruit and dried under shade. Kernels were separated from dried seeds, powdered and stored in air tight containers for further studies. Mango kernel powders were extracted for phytochemicals by using different organic solvents $(50 \mathrm{~mL})$ like methanol, ethanol, acetone, chloroform and ethyl acetate. Dried BK, MK, TK and NK powders were weighed (5 gms) into different conical flasks and were extracted overnight with methanol $(50 \mathrm{~mL})$ in a shaking incubator. Extracts of four kernels were filtered through whatman No.1 filter paper into different conical flasks [8]. The procedure was repeated for three consecutive days and the filtrate was pooled up and was concentrated under vacuum in a Heidolph Rota evaporator at $40{ }^{\circ} \mathrm{C}$. The concentrates were stored in amber coloured bottles under refrigerated conditions until further studies. Similar procedure was adapted to extract the kernels by using remaining four solvents.

\subsection{Extraction Efficiency and Total Phenolic Content}

BK, MK, TK and NK were extracted with five different organic solvents to know the extraction efficiency of the solvent and concentration of TPC in different extracts [9]. The extracts of each solvent were tested for TPC [10]. Extract (0.2 mL) was mixed with 1 $\mathrm{mL}$ of 10 fold diluted Folin-ciocalteu reagent and 0.8 $\mathrm{mL}$ of $7.5 \%$ sodium carbonate solution. After standing for 30 minutes at room temperature, the absorbance was measured at $765 \mathrm{~nm}$ using UV-visible spectrophotometer-160 A (Shimadzu, Japan). The extract which showed highest phenolic concentration was used for further studies.

\subsection{Free Radical Scavenging Ability by Using a Stable DPPH Radical}

This is the most widely reported method for screening of AA of many bioactive compounds. DPPH assay method is based on the reduction of methanolic solution of colored free radical DPPH, which is proportional to concentration of free radical scavenger added to DPPH reagent solution. The DPPH radical scavenging activity of kernel extracts was determined using the method proposed by Hansmann [11]. Methanolic solutions of synthetic antioxidants were also tested at different concentrations. BHT and butylated hydroxyl anisole (BHA) solutions were used as synthetic antioxidants. The percentage inhibition of the DPPH radical by the samples was calculated according to the formula of Yen and Duh [12].

\%inhibition $=\left[\left(\mathrm{A}_{\mathrm{C}(\mathrm{O})}-\mathrm{A}_{\mathrm{A}(\mathrm{t})} / \mathrm{A}_{\mathrm{C}(\mathrm{O})}\right] \times 100\right.$

where $\mathrm{A}_{\mathrm{C}(\mathrm{O})}$ is the absorbance of the control and $t=0$ $\min$;

and $\mathrm{A}_{\mathrm{A}(\mathrm{t})}$ is the absorbance of the antioxidant at $t=16$ $\min$.

\subsection{Relative Reducing Power (RRP)}

This method is based on the principle of increase in the absorbance of the reaction mixture [13]. In this, antioxidant compound forms a colored complex with potassium ferricyanide, trichloroacetic acid and ferric chloride, which is measured at $700 \mathrm{~nm}$. Increase in absorbance of the reaction mixture, indicates the reducing power of the samples. The reducing power of 
the extracts was measured as described by Oyaizu [14]. Relative reducing power of the extracts was calculated by the equation.

$\mathrm{RRP}=($ Sample absorption at $700 \mathrm{~nm} / 1.2) \times 100(2)$

\subsection{HPLC Identification of Phenolic Compounds}

Extracts were subjected to HPLC analysis by a slight modification [15] proposed by Mi-Jung Kim, using Shimadzu LC-20AD pumps, SPC-M20 with diode array detector and chromatographic separations were performed on a Xtimate C-18 column $(4.6 \times 250 \mathrm{~mm}$ i.d., $5 \mu \mathrm{m})$. The mobile phase was $0.2 \%$ glacial acetic acid in water as solvent $\mathrm{A}$ and acetonitrile as solvent $\mathrm{B}$. The gradient programme was $0 \mathrm{~min} 5 \% \mathrm{~B}$ in $\mathrm{A} ; 60 \mathrm{~min}$ $30 \% \mathrm{~B}$ in A with flow rate of $1 \mathrm{~mL} / \mathrm{min}$. Column temperature was $25{ }^{\circ} \mathrm{C}$; injection volume of sample was $20 \mu \mathrm{L}$ and photo diode array detection was carried at $275 \mathrm{~nm}$. The compounds were identified from their peak areas in relation to respective reference standards.

\subsection{Treatment of RBD-SBO with Mango Kernel Extracts or Synthetic Antioxidant}

RBD SBO free from additives was used as substrate for oxidation studies. RBD SBO was weighed in different amber coloured bottles. These oil samples were treated with baneesha kernel extract (BKE), malgoa kernel extract (MKE), totapuri kernel extract (TKE) and neelam kernel extract (NKE) at the rate of $200 \mathrm{ppm}$ or with synthetic antioxidant BHT at the same concentration and tested for comparative purposes at their legal limit of $200 \mathrm{ppm}$ as specified [16, 17]. Control sample of RBD SBO without any antioxidant was also prepared and bottled under identical conditions.

\subsection{AA of Mango Kernel Extracts in RBD SBO}

AA of different kernel extracts in RBD SBO was investigated by two methods, the Rancimat method [18] and by estimating the peroxide value (PV) of the oil samples which are heated at different temperatures $\left(40{ }^{\circ} \mathrm{C}-130{ }^{\circ} \mathrm{C}\right)$ in hot air-oven.

\subsubsection{Rancimat Method}

Metrohm Rancimat model 743 capable of operating over a range of $50{ }^{\circ} \mathrm{C}-220^{\circ} \mathrm{C}$ was used for knowing the induction period of the oil. A stream of air was bubbled into oil samples contained in the reaction vessel placed in an electric heating block. Effluent air containing volatile organic acids from the oil samples were collected in measuring vessel containing distilled water $(60 \mathrm{~mL})$. The conductivity of the water was measured automatically as oxidation proceeded. Filtered, cleaned, dried air was allowed to bubble through the hot oil at rates of $20 \mathrm{~L} / \mathrm{h}$. A $5 \mathrm{gm}$ portion of RBD SBO with different extracts or synthetic antioxidant BHT and control (without any additive) were loaded into reaction vessels for carrying out the experiment. The induction period was estimated at $130{ }^{\circ} \mathrm{C}$ and was recalculated at different temperatures i.e., from $40{ }^{\circ} \mathrm{C}-120^{\circ} \mathrm{C}$ in the rancimat.

\subsubsection{Hot Air Oven Method}

A known portion of each sample was weighed in a glass cup and heated in a hot air oven for one hour at different temperatures $\left(40{ }^{\circ} \mathrm{C}-130{ }^{\circ} \mathrm{C}\right)$. Fresh sample was taken for each treatment. The PV (expressed as milli equivalents of peroxide per 1,000 gms of oil/fat) of RBD SBO after each treatment was determined by AOCS [19] official method Cd8-53.

\subsection{Statistical Analysis}

All determinations were carried out in triplicate. Statistical analyses were performed by using Microsoft Excel Data Analysis. Significant differences between means were determined by Duncan's multiple range tests [20] and were considered to be significant when $P<0.05$.

\section{Results and Discussion}

\subsection{Extraction Efficiency and TPC}

The yield of extracts obtained from BK, MK, TK and NK using five different organic solvents independently (i.e., methanol, ethanol, acetone, chloroform and ethyl acetate) is shown in Fig. 1. Methanol gave maximum yield of extract for all kernels. 


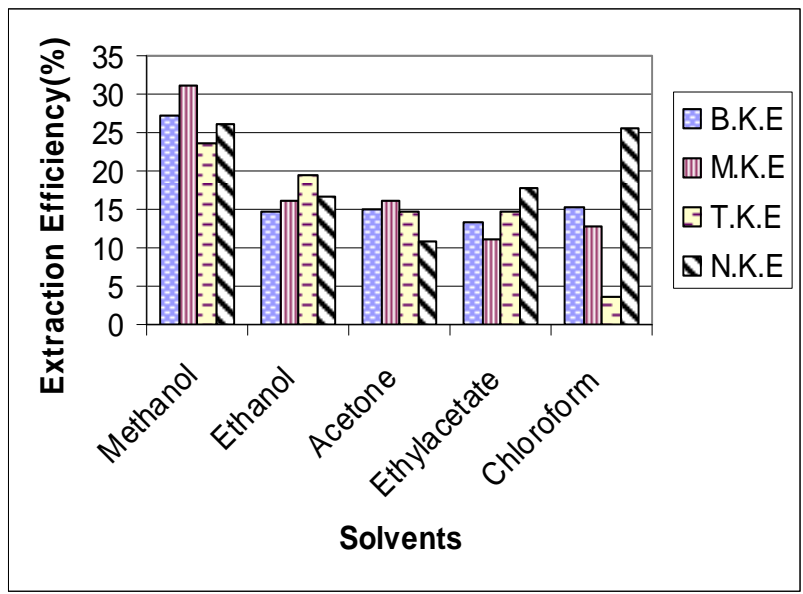

Fig. 1 Extraction efficiency (\%) of different solvents with mango kernels.

It was observed that methanol was the best solvent among the five solvents used in the extraction of mango kernels. All other solvents also gave significant yields with four mango kernels except chloroform with TK.

Extracts of all the five solvents were tested for TPC by Folin-Ciocalteau method spectrophotometrically and results are shown in Fig. 2. It clearly indicates that the amount of TPC in the methanol extract was the highest. So, methanol extracts of all four kernels were used for further analysis.

Methanol extracts of all kernels gave maximum TPC. MKE had highest TPC (88.33) followed by TKE (87.75), NKE (65.29) and BKE (63.42). Ethanol extracts showed significant TPC from BK and NK. The remaining three solvents showed low TPC from all mango kernels under study. The present study indicates that methanolic extracts of mango varieties contain expressive TPC that may contribute to AA. In a study [21] the TPC of mango kernels were in the range of 70 to $200 \mathrm{mg} / \mathrm{kg}$. TPC of mango kernel extracts in the present study is far higher than the TPC of Egyptian varieties reported [22] and were lower than TPC of organically grown Uba variety mangoes [23]. The variation in TPC of mango kernels of present study from previous study may be due to varietal differences, difference in the nature of raw material and method of extraction.

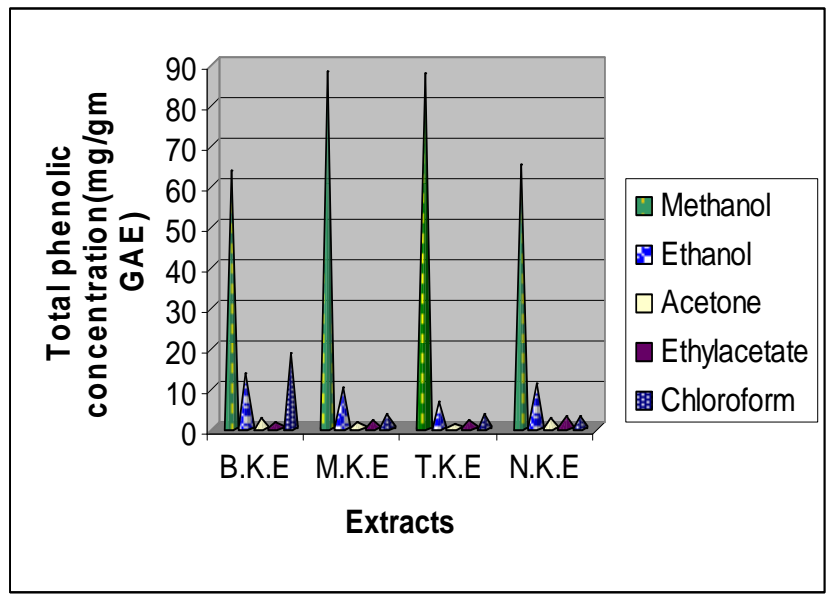

Fig. 2 TPC (mg/gm GAE) of different mango kernel extracts.

\subsection{DPPH Activity}

Free radical scavenging potential of BKE, MKE, TKE and NKE at four different concentrations (5, 25, 50 and $100 \mathrm{ppm}$ ) was tested by the DPPH method. The degree of discoloration indicates the scavenging potentials of the antioxidant extract. The DPPH activity of extracts is shown in Fig. 3. The activities of the extracts were compared with BHA and BHT. At all concentrations, BHA showed the least activity when compared with others. The RSA of BKE, MKE, TKE, NKE, BHT and BHA at $100 \mathrm{ppm}$ is $85 \%, 89 \%, 89 \%$, $84 \%, 80 \%$ and $78 \%$ respectively. All kernel extracts exhibited a dose dependent free RSA. This activity of mango kernel extracts can be attributed to its hydrogen donating ability [24]. MKE and TKE showed a higher RSA than BKE and NKE. This may be due to higher phenolic concentration of those two extracts. DPPH activity can be expressed in the following manner to show their increased order of activity.

$$
\mathrm{BHA}<\mathrm{BHT}<\mathrm{NKE}<\mathrm{BKE}<\mathrm{TKE}=\mathrm{MKE}
$$

Mango kernel extracts of Thai varieties also showed a higher DPPH activity than vitamin $\mathrm{C}, \mathrm{BHA}$ and Trolox [25]. RSA of mango kernel extracts was dose dependent in this study which can be correlated with an earlier work [23].

\section{$3.3 R R P$}

Mango kernel extracts were tested for RRP at five different concentrations $(50,100,250,500,1000$ ppm) 


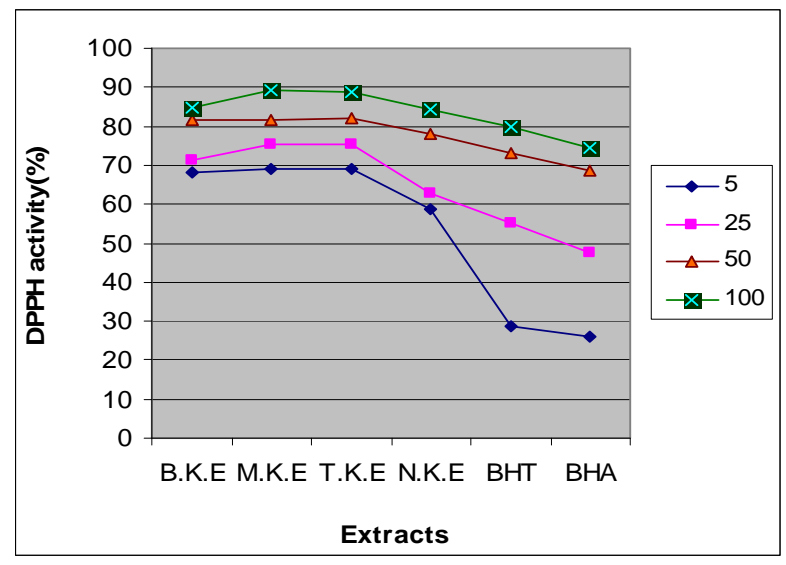

Fig. 3 DPPH activity (\%) of mango kernel extracts in comparison with BHA and BHT.

and the results are shown in Fig. 4. The RRP is found to be dependent on extract concentration. There was a gradual increase in the reducing power from 50 to 1000 ppm. As more $\mathrm{Fe}^{3+}$ are reduced to the ferrous form or when more electrons are donated by antioxidant components the colour of Pearl's Prussian Blue will be darker, resulting in an increased absorbance readings [26].

BKE, MKE, NKE and TKE showed RRP of $76 \%$, $82 \%, 80 \%$ and $66 \%$ respectively at 1,000 ppm levels. The presence of RRP indicates that the mango kernel extracts have electron donors and can react with free radicals to convert them to more stable products and terminate radical chain reaction. The highest RRP of the MKE is in correlation with its TPC.

Aqueous methanolic extracts of Uba variety mangoes showed a higher RSA and reducing power [23] than BHA at 1,000 ppm where as mango kernel extracts in the present study showed a higher activity than BHA and BHT at a lowest concentration.

The different antioxidant potentials of mango varieties found in this work can be attributed, not only to genotype differences [27] but also to varied agricultural practices [28].

\subsection{Identification of Phenolic Compounds}

Mango kernel extracts were subjected to HPLC for identification of phenolic compounds and their chromatograms are given in Figs. 5-8. TPC of mango

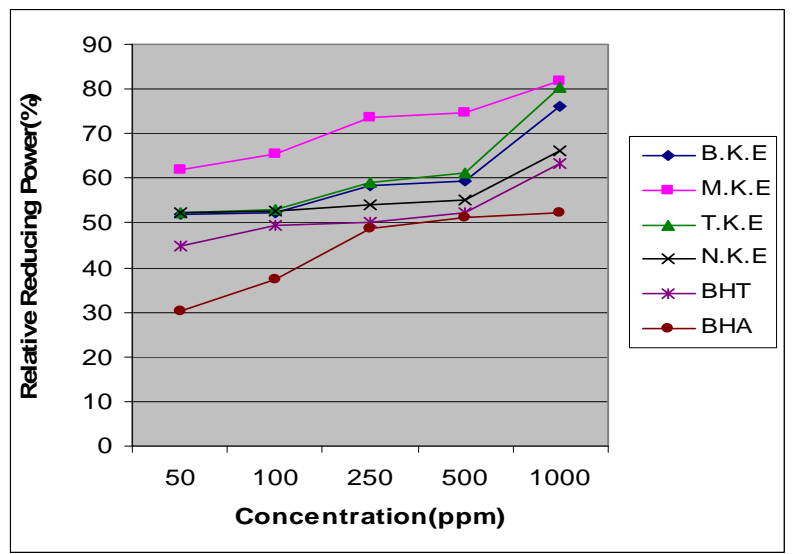

Fig. 4 RRP (\%) of mango kernel extracts in comparison with BHA and BHT.

kernel extracts was expressed as Gallic acid equivalents; hence the Gallic acid peak of these extracts is shown separately.

Mango kernel extracts showed the presence of different concentrations of phenolic compounds which may be the main attribute for the change in their AA. From the Table 1, it can be noted that MKE showed the highest concentration of identified phenolic compounds. MKE (886.05) and TKE (265) showed the presence of kaempferol in high concentration but quercetin was not detected in these extracts. BKE had tocopherol (354.66); both MKE (549.7) and NKE (452.15) had rutin in high concentration.

HPLC analysis of mango kernel extracts in the present study is in correlation with a previous study which showed the presence of tocopherols and other phenolic compounds. Abdalla et al. [29] characterized phenolic compounds in Egyptian mango kernels in which the components included were tannins, Gallic acid, coumarin, ellagic acid, vanillin, mangiferin, ferulic acid, cinnamic acid and unknown compounds.

RBD SBO samples were tested in Rancimat by incorporating all four extracts, synthetic antioxidant (BHT) separately and control to know the induction period. The RBD SBO samples with MKE and TKE showed a higher IP at all temperatures i.e., from 40 to $130{ }^{\circ} \mathrm{C}$ than RBD SBO samples with other extracts, BHT and control when tested in Rancimat. The IP of RBD SBO samples with BKE, MKE, TKE, NKE, BHT 


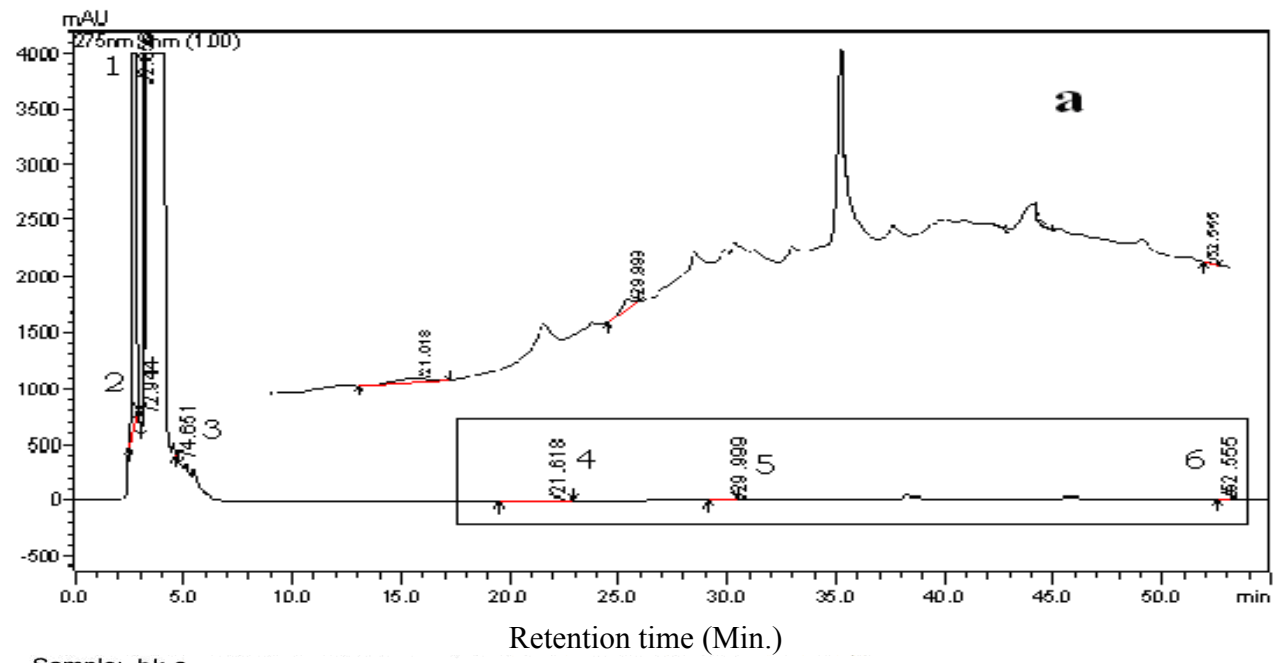

Sample: -bk-s

Scan Range: 1 - 356 Time Range: $0.00-4.98 \mathrm{~min}$

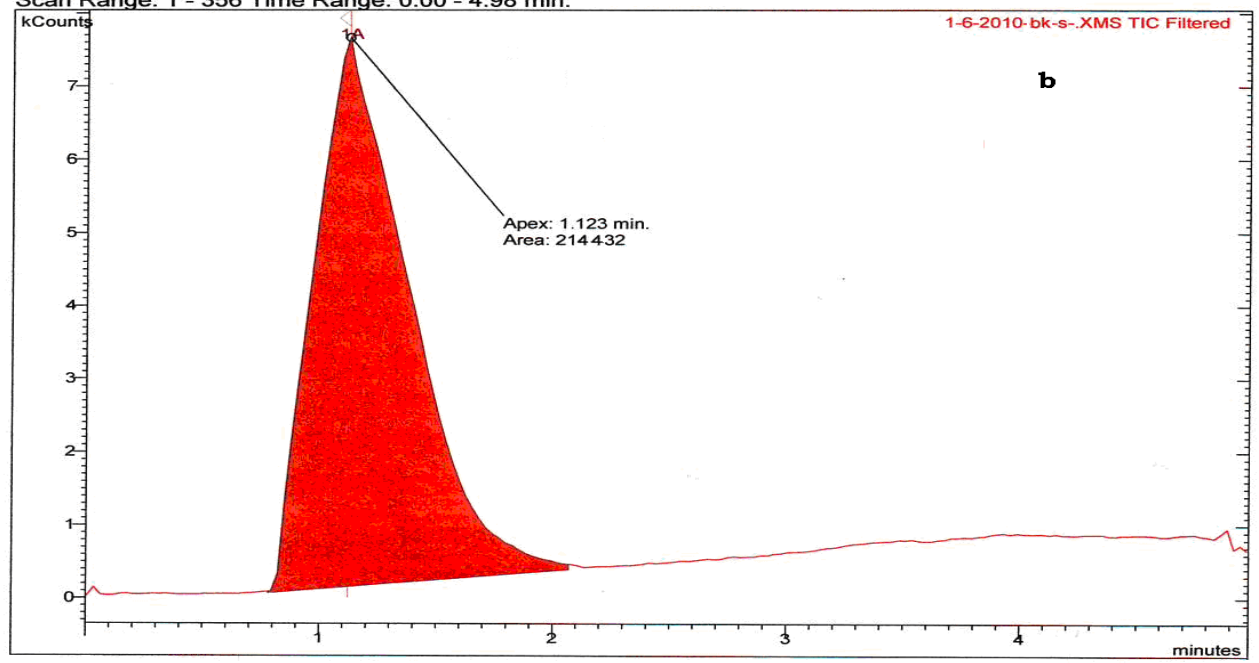

Retention time (Min.)

Fig. 5 Chromatogram of phenolic compounds in BKE.

Part a (1) $\alpha$-tocopherol; (2) rutin; (3) kaempferol; (4) vanillic acid; (5) ferulic acid; (6) quercetin. Peaks 4, 5, 6 of the graphs are magnified. (b) Magnified Gallic acid peak.

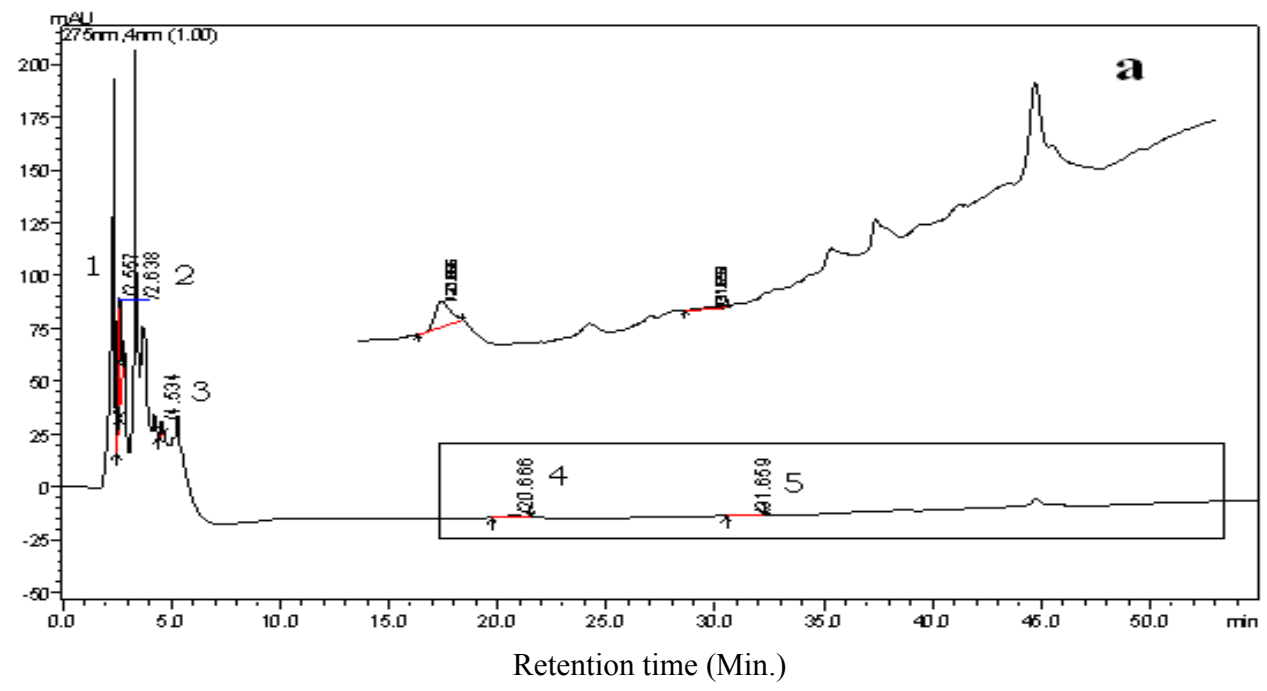




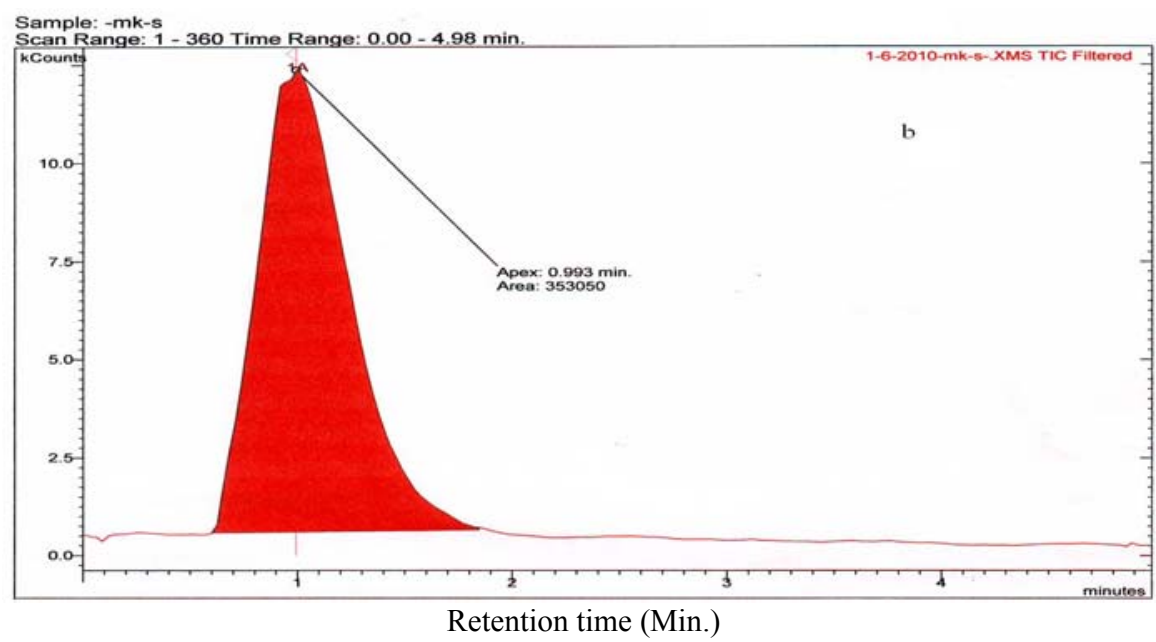

Fig. 6 Chromatogram of phenolic compounds present in MKE.

Part a (1) $\alpha$-tocopherol; (2) rutin; (3) kaempferol; (4) vanillic acid; (5) ferulic acid; Peaks 4, 5 of the graphs are magnified. (b) Magnified Gallic acid peak.

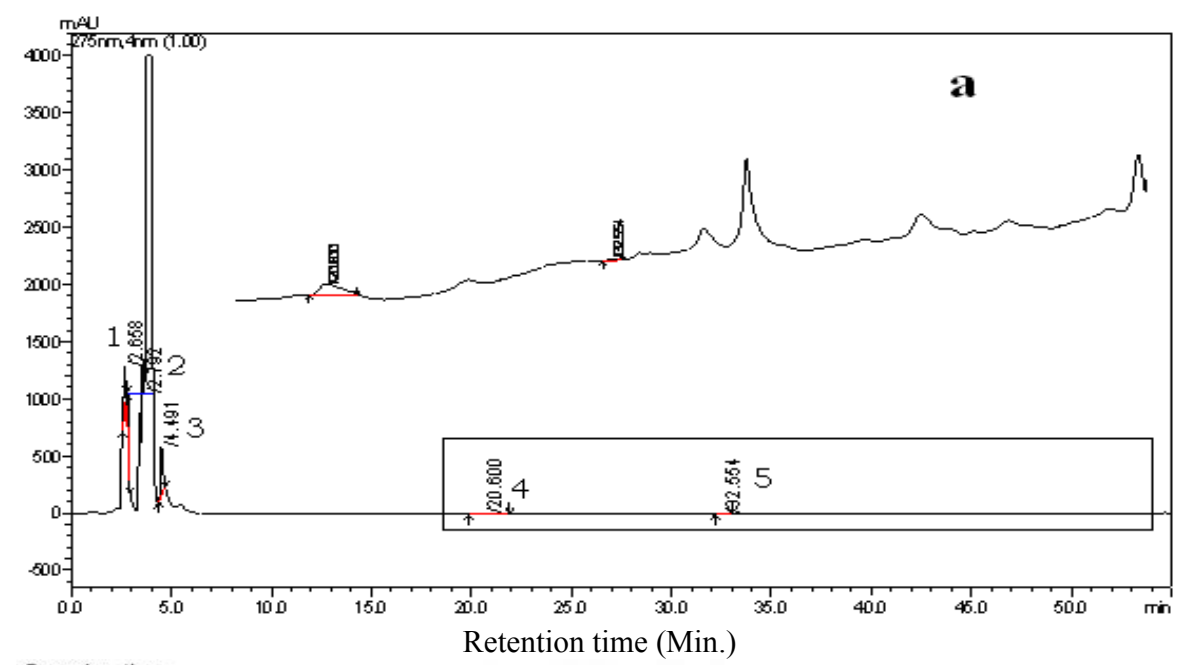

Sample: -tk-s-

Scan Range: 1 - 357 Time Range: $0.00-4.99 \mathrm{~min}$.

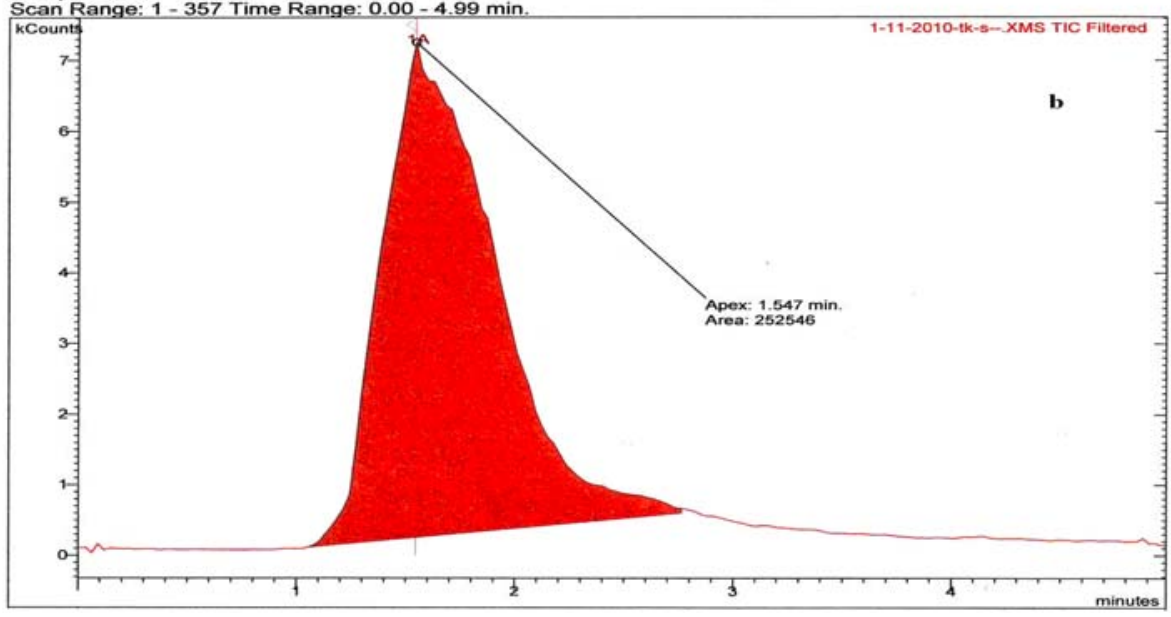

Retention time (Min.)

Fig. 7 Chromatogram of phenolic compounds present in TKE.

Part a (1) $\alpha$-tocopherol; (2) rutin; (3) kaempferol; (4) vanillic acid; (5) ferulic acid. Peaks 4, 5 of the graphs are magnified. (b) Magnified Gallic acid peak. 


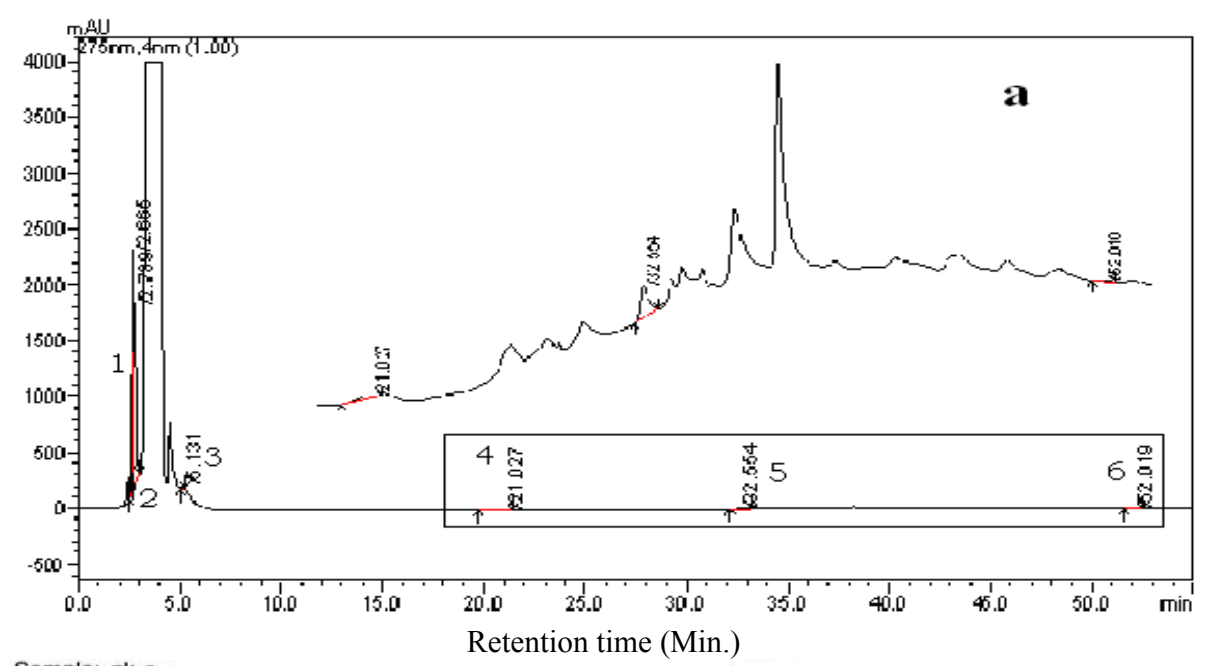

Sample:-nk-s-

Scan Range: 1 - 355 Time Range: 0.00 - $4.98 \mathrm{~min}$

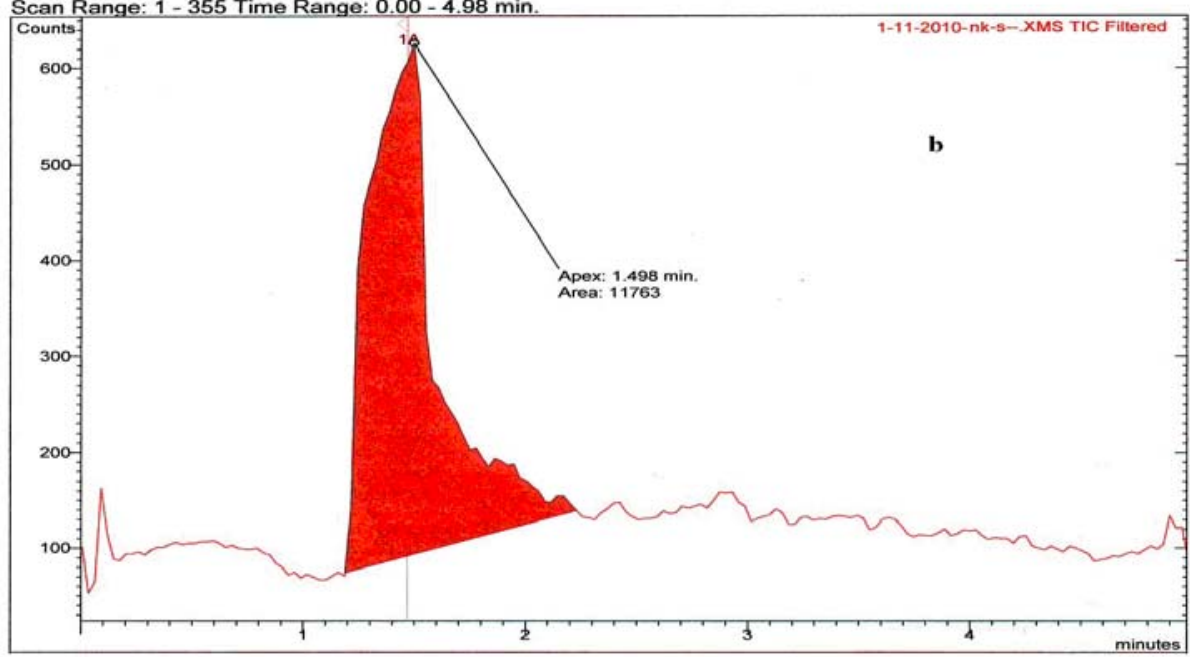

Retention time (Min.)

Fig. 8 Chromatogram of phenolic compounds present in NKE.

Part a (1) $\alpha$-tocopherol; (2) rutin; (3) kaempferol; (4) vanillic acid; (5) ferulic acid; (6) quercetin. Peaks 4, 5, 6 of the graphs are magnified. (b) Magnified Gallic acid peak.

Table 1 Quantification of phenolic compounds in mango kernel extracts.

\begin{tabular}{lcccc}
\hline \multirow{2}{*}{ Compound } & \multicolumn{3}{c}{ Concentration $(\mathrm{mg} / 100 \mathrm{~g})$} \\
\cline { 2 - 5 } & BKE & MKE & TKE & NKE \\
\hline a-Tocopherol & 354.66 & 90.35 & 3.975 & 93.35 \\
Vanillic acid & 0.5 & 15.54 & 11.725 & 0.145 \\
Ferulic acid & 44.5 & 1.08 & 0.06 & 0.58 \\
Rutin & 0.04 & 549.7 & 139.8 & 452.15 \\
Kaempferol & 22.465 & 886.05 & 265 & 74.335 \\
Quercitin & 1.4 & ND & ND & 0.039 \\
Gallic acid & 43.77 & 176.75 & 163.92 & 23.45 \\
\hline
\end{tabular}

ND: not detected.

and control at $130^{\circ} \mathrm{C}$ is $2.28,2.31,2.31,2.01,0.85$ and 0.53 respectively. The results of IP period of RBD SBO samples are shown in Fig. 9.
The data on the effect of the kernel extracts to prevent lipid peroxidation are shown in Fig. 10. All the kernel extracts were effective in preventing the peroxide 


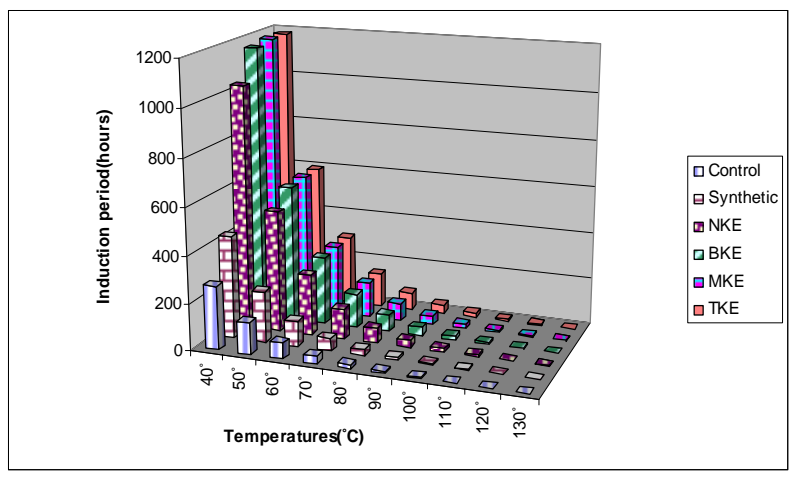

Fig. 9 IP of RBD SBO with mango kernel extracts.

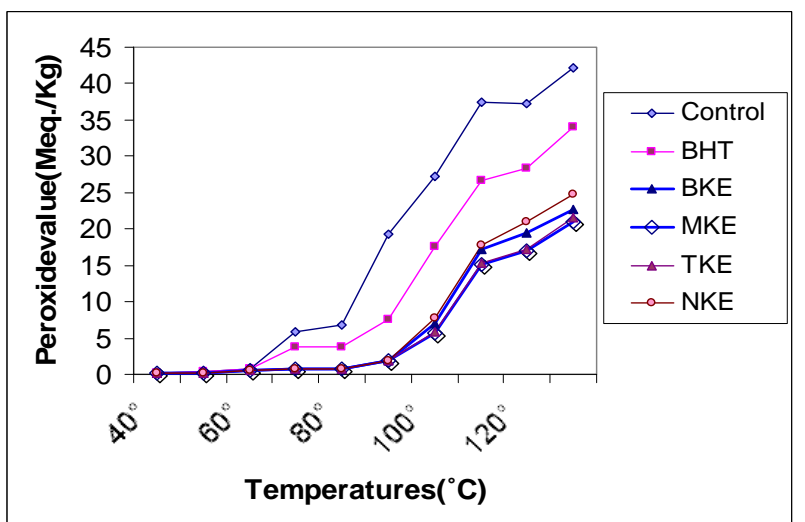

Fig. 10 PV of RBD SBO with mango kernel extracts in comparison with BHT.

formation of RBD SBO than synthetic antioxidant at the same concentration. When RBD SBO samples were heated to different temperatures and tested for PV, the RBD SBO samples with MKE showed a lower PV (20.96) than RBD SBO samples containing BKE (23), TKE (21.54), NKE (24), BHT (34.09) and RBD SBO samples without any additive $(42.16)$ at $130{ }^{\circ} \mathrm{C}$.

The stability of the RBD SBO samples to the formation of peroxides and the induction period can be ranked in the following descending order;

Peroxide value Control $>$ BHT $>$ NKE $>$ BKE $>$ TKE $>$ MKE

IP $\mathrm{MKE}=\mathrm{TKE}>\mathrm{BKE}>\mathrm{NKE}>\mathrm{BHT}>$ Control

The polyphenols present in all the four extracts have potential contribution to $\mathrm{AA}$ and prevention of rancidity in RBD SBO samples. Phenolic antioxidants may have synergistic activity. The high AA of the mango kernel extracts appeared to be attributed to its high phenolic content.
Earlier studies [30] on AA of mango kernel extracts showed that they were able to maintain the oxidative stability of ghee. The present study shows that mango kernel extracts are having the capacity to prevent rancidity of poly unsaturated fatty acids in SBO.

\section{Conclusions}

The present study shows that kernels of four different varieties of mangoes possessed AA which is shown by different in vitro tests i.e., radical scavenging activity and RRP. HPLC analysis of extracts showed that they had different phenolic compounds like Gallic acid, tocopherol, vanillic acid, rutin, ferulic acid, kaempferol and quercitin. MKE and TKE showed a higher AA than other two kernel extracts which is in correlation to their TPC. When tested in RBD SBO, the extracts had a capacity to slow down the formation of peroxides and increase induction period than BHT at the same concentration. Mango kernel extracts may be used as a source of natural antioxidant at industrial level to reduce the health hazards caused by synthetic antioxidants. We consider that these mango kernel extracts deserve more intense study including its antioxidant composition, their bio-availability and possible protection against diseases. Further, studies into the absorption and effect of the extracts of BK, MK, TK and NK on antioxidant status in animal models are needed to evaluate their potential health benefits.

AA of mango kernel extracts had a positive correlation with their TPC.

\section{Acknowledgments}

The authors are thankful to the JNT University authorities for their support and co-operation. The authors are also grateful to the Director, JNTUA OTRI, Anantapur for his support and cooperation and Head, Lipid Science Dept. I.I.C.T. Hyderabad for allowing to work on Rancimat. Special thanks to Dr. Md. Akthar, S.K University, Anantapur for his technical help in Statistical analyses. 


\section{References}

[1] K. Nagendra Prasad, Soundar Divakar, Gyarahally R. Shivamurthy, Somaradhya mallikarjuna aradhya, Isolation of a free radical-scavenging antioxidant from water spinach (Ipomea aquatica Forsk), J. Sci. Food Agric. 85 (2005) 1461-1468.

[2] Sonia, Antioxidant in mango (Mangifera indica L.) pulp, Plant Foods for Human Nutrition 62 (2007) 13-17.

[3] S. Okonogi, C. Duangrat, S. Anuchpreeda, S. Tachakittirungrod, S. Chowwanapoonpohn, Comparison of antioxidant capacities and cytotoxicities of certain fruit peels, Food Chem.103 (2007) 839-846.

[4] I. Amin, O. Mukhrizah, Antioxidant capacity of methanolic and water extracts prepared from food-processing by-products, J. Sci. Food Agric. 86 (2006) 778-784.

[5] S.S. Parmar, R.S. Sharma, Effect of mango (Mangifera indica L.) seed kernels pre-extract on the oxidative stability of ghee, Food Chem. 35 (1990) 99-107.

[6] S.S. Arogba, Mango (Mangifera indica) kernel, Chromatographic analysis of the tannin and stability of the associated polyphenol oxidase activity, J. Food Comp. Anal. 13 (2000) 149-156.

[7] D. Puravankara, V. Boghra, R.S. Sharma, Effect of antioxidant principles isolated from mango (Mangifera indica $\mathrm{L}$ ) seed kernels on oxidative stability of buffalo ghee (butter- fat), J. Sci. Food Agric. 80 (2000) 522-526.

[8] P.D. Duh, D.B. Yeh, G.C. Yen, Extraction and identification of an antioxidative component from peanut hulls, J. Am. Oil Chem. Soc. 69 (1992) 814-818.

[9] G.C. Yen, P.D. Duh, Antioxidant activity of methanolic extracts of peanut hulls from various cultivars, J. Am. Oil Chem. Soc. 72 (1995) 1065-1067.

[10] J. Perez-Jimenez, F. Saura-Calixto, Effect of solvent and certain food constituents on different antioxidant capacity assays, Food Res. Intern. 39 (2006) 791-800.

[11] A. Von Gadow, E. Joubert, C.F. Hansmann, Comparison of antioxidant activity of aspalathin with that of other plant phenols of Robioosed tea (Aspalathon linearis), $\alpha$-tocopherol, BHT and BHA, J. Agric. Food Chem. 45 (1997) 632-638.

[12] G.C. Yen, P.D. Duh, Scavenging effect of methanolic extracts of peanut hulls on free-radical active oxygen species, J. Agric. Food Chem. 42 (1994) 629-632.

[13] B.V. Ramakrishna, G.K. Jayaprakasha, B.S. Jena, R.P. Singh, Antioxidant activities of roselle (Hibiscus sabdariffa) calyces and fruit extracts, J. Food Sci. Technol. 45 (2008) 223-227.

[14] M. Oyaizu, Studies on products of the browning reaction. Antioxidative activities of products of browning reaction prepared from glucosamine, Jap. J. Nutr. 44 (1986) 307-315.

[15] A. Schieber, N. Berardini, R. Carle, Identification of flavonol and xanthone Glycosides from mango (Mangifera indica L. Cv. "Tommy Atkins") peels by High-Performance Liquid Chromatography-Electro spray Ionization Mass Spectrometry, J. Agric. Food Chem. 51 (2003) 5006-5011.

[16] G.C. Yen, P.D. Duh, Scavenging effect of methanolic extracts of peanut hulls on free-radical active oxygen species, J. Agric. Food Chem. 42 (1994) 629-632.

[17] Prevention of food adulteration Act of 1954, Prevention of Food Adulteration, in: A.P. Mathur (Ed.), $7^{\text {th }}$ ed., Eastern Book Company, Lucknow, 1976, p. 662.

[18] Reza Farhoosh, The effect of operational parameters of the Rancimat method on the determination of the oxidative stability measures and shelf-life prediction of soybean oil, J. Am. Oil Chem. Soc. 84 (2007) 205-209.

[19] A.O.C.S., Official Methods and Recommended Practices of the American Oil Chemists' Society, $5^{\text {th }}$ ed., Cd 8-53 A.O. C.S. press, Champaign, IL, USA, 1998.

[20] B. Duncan, Multiple range and multiple $f$ test, Biometrics 11 (1955) 1-5.

[21] Jacqueline C. Barreto, Maria T.S. Trevisan, William E. Hull, Gerhard Erben, Edy S. DE Brito, Beate Pfudstein, et al., Characterization and quantification of polyphenolic compounds in bark, kernel, leaves and peel of Mango (Mangifera indica L.), J. Agric. Food Chem. 56 (2008) 5599-5610.

[22] I.S. Ashoush, M.G.E. Gadallah, Utilization of mango peels and seed kernels powders as sources of phytochemicals in biscuit, W. J. Dairy and Food Sci. 6 (2011) 35-42.

[23] S.M.R. Ribeiro, L.C.A. Barbosa, J.H. Queiroz, M. Knodler, A. Schieber, Phenolic compounds and antioxidant capacity of Brazilian mango (Mangifera indica L.) varieties, Food Chem. 110 (2008) 620-626.

[24] K. Shimada, K. Fujikawa, K. Tahara, T. Nakamura, Antioxidative properties of xanthan on the autoxidation of soybean oil in cyclodextrin emulsion, J. Agric. Food Chem. 40 (1992) 945-948.

[25] Saranyu Khammuang, Rakrudee Saranthima, Antioxidant and antibacterial activities of selected varieties of Thai Mango seed extract, Pak. J. Pharm. Sci. 24 (2011) 37-42.

[26] L.S. Lai, S.T. Chou, W.W. Chao, Studies on the antioxidative activities of Hsian-tsao (Mesona procumbans Hesml) leaf gum, J. Agric. Food Chem. 49 (2001) 963-968.

[27] J. Scalzo, Politi, A Pellegrini, B. Mezzetti, M. Batino, Plant genotype affects total antioxidant capacity and phenolic content in fruit, Nutrition 21 (2005) 207-213. 
[28] S.Y. Wang, W. Zheng, G.J. Galletta, Cultural system affects fruit quality and antioxidant capacity in strawberries, Journal of Agric and Food Chem. 50 (2002) 6534-6542.

[29] A.E.M. Abdalla, S.M. Darwish, E.H.E. Ayad, R.M. El-Hamahmy, Egyptian mango by-product: 1.
Compositional quality of mango seed kernel, Food Chemistry 103 (2007) 1134-1140.

[30] D. Purvankara, V. Boghra, R.S. Sharma, Effect of antioxidant principles isolated from mango (Mangifera indica L.) seed kernels on oxidative stability of buffalo ghee (butter-fat), J. Sci. Food Agric. 80 (2000) 522-526. 